\title{
Relapsed/Refractory Diffuse Large B-Cell Lymphoma and Mantle Cell Lymphoma: Is It Time to Consider CAR-T for All?
}

\author{
Presented by Andrew D. Zelenetz, MD, PhD
}

\begin{abstract}
CAR T cells have demonstrated activity in relapsed/refractory non-Hodgkin lymphoma, including diffuse large B-cell lymphoma (DLBCL), indolent non-Hodgkin lymphoma, and mantle cell lymphoma. For patients with chemorefractory disease, CAR T cells can provide a durable complete response in a portion of patients, which represents a major advance in the field. For patients with chemosensitive disease, however, additional data are needed to determine whether CAR T cells are preferable to conventional approaches. Studies in DLBCL have shown that patients experiencing a PET-positive partial response after second-line chemotherapy have long-term outcomes after high-dose therapy and autologous stem cell rescue that are similar to CAR T-cell therapy, with decreased toxicity and cost. Alternative third-line options such as tafasitamab/ lenalidomide and bispecific antibodies may also have a role for patients with chemorefractory disease.

J Natl Compr Canc Netw 2020;18(12.5):1764-1766
\end{abstract} doi: $10.6004 /$ jnccn.2020.5036

CAR T cells continue to show impressive activity in relapsed/refractory non-Hodgkin lymphoma, with durable complete responses in a significant percentage of patients with chemorefractory disease. According to Andrew D. Zelenetz, MD, PhD, Medical Director of Quality Informatics, Memorial Sloan Kettering Cancer Center, and Chair of the NCCN Non-Hodgkin Lymphoma Panel, however, for patients with chemosensitive, there is still debate about whether CAR T cells are superior to conventional approaches. At the NCCN 2020 Virtual Congress: Hematologic Malignancies, Dr. Zelenetz discussed the role of CAR $T$ cells in diffuse large B-cell lymphoma (DLBCL) and mantle cell lymphoma.

\section{Diffuse Large B-Cell Lymphoma}

Axicabtagene Ciloleucel

Axicabtagene ciloleucel was approved by the FDA for the treatment of DLBCL in 2018 based on results of the phase II ZUMA-1 trial, which included 77 patients with refractory DLBCL and 24 patients with either transformed lymphoma or refractory primary mediastinal B-cell lymphoma. ${ }^{1}$ As Dr. Zelenetz reported, this was a population with heavily refractory disease. Nearly $70 \%$ of patients had received $\geq 2$ prior treatments, $26 \%$ had primary refractory disease, and half of patients had disease resistant to 2 consecutive lines of treatment.

Every patient on trial experienced some form of adverse event, said Dr. Zelenetz. He noted that $8 \%$ of patients died due to toxicity, and almost $90 \%$ experienced grade 3 or 4 treatment-related adverse events.

Despite the toxicity, however, this has been a highly effective treatment, he said. The objective response rate by the independent review committee was $74 \%$, with a complete response rate of $54 \%$. The most recent followup showed an ongoing response of $36 \%$, and the median response duration has still not been reached in the independent review committee assessment.

"One of the most striking features is that after the rapid drop-off in the first 3 months, the curves for both progression-free survival (PFS) and response duration are quite stable," said Dr. Zelenetz, who noted that expected overall survival (OS) for this group of patients would be between $10 \%$ and $15 \%$. "Median [OS] in this study was not reached."

According to Dr. Zelenetz, patients who experience a complete or partial response have excellent PFS, whereas those with stable disease tend to have recurrence after treatment with these agents. Patients with an ongoing response on axicabtagene ciloleucel also had a significantly greater peak T-cell expansion in peripheral blood compared with those who experienced progression or had no response to CAR T cells.

\section{Tisagenlecleucel}

Tisagenlecleucel, a 4-1BB construct targeting CD19, was approved based on data from the JULIET trial $(n=111){ }^{2}$ This study cohort consisted of a relatively young population of patients, said Dr. Zelenetz, but again represented 
poor-risk patients, with $>50 \%$ of patients having $\geq 3$ lines of treatment and/or a history of refractory DLBCL. Although safety data showed that $100 \%$ of patients experienced some adverse event, rates of grade 3 and 4 cytokine-release syndrome were relatively low, Dr. Zelenetz reported. The risk of neurologic events was also somewhat lower than that observed with axicabtagene ciloleucel.

"The best overall response of $52 \%$, with a complete response rate of $40 \%$, was a little bit lower than axicabtagene," said Dr. Zelenetz, who acknowledged that cross-trial comparisons are hazardous.

According to Dr. Zelenetz, although excellent response was seen in all groups, patients whose disease was refractory to their immediate prior line of therapy tended to have a poor response. "Not surprisingly, patients with a complete response have a better response duration and PFS compared with the entire population, but just like we saw with axicabtagene ciloleucel, those responses were durable regardless of the depth of response," said Dr. Zelenetz. "Conversely, patients with stable disease or failure to respond on treatment did poorly."

\section{Lisocabtagene Maraleucel}

Lisocabtagene maraleucel (liso-cel) is another CD19directed CAR $\mathrm{T}$ cell that uses the 4-1BB costimulatory domain. Unlike tisagenlecleucel, however, liso-cel is a defined composition product. CD8 and CD4 T cells are independently transduced and returned to the patient in a 1-to-1 ratio.

The FDA is currently considering approval of liso-cel based on data from the TRANSCEND trial, which enrolled a heavily pretreated population of patients. ${ }^{3} \mathrm{Ap}-$ proximately $50 \%$ of patients had $\geq 3$ prior lines of therapy, and two-thirds were refractory to chemotherapy. As Dr. Zelenetz reported, the rate of grade 3 and 4 cytokinerelease syndrome was very low at only $2 \%$. Grade 3 and 4 neurologic events were also relatively low, with only $10 \%$ of patients experiencing grade 3 and 4 toxicity.

"As this was the third anti-CD19 CAR T cell, many centers had more experience in managing cytokinerelease syndrome," said Dr. Zelenetz. He noted that $20 \%$ of patients received tocilizumab and/or corticosteroids for cytokine-release syndrome. "The first use of tocilizumab was often given when patients had only grade 1 or 2 toxicity to try to prevent the development of grade 3 and 4 toxicity."

The objective response rate was $73 \%$, including a complete response rate of $53 \%$ and a partial response rate of $20 \%$, and these response were observed across different subtypes. According to Dr. Zelenetz, patients with very bulky disease were the only group that did not respond well, suggesting a role for pretreatment bridging or cytoreductive therapy. Although patients with partial responses did not fare as well, those with complete responses have done "extremely well" with respect to duration of response, said Dr. Zelenetz.

\section{High-Dose Therapy With Autologous Stem}

Cell Rescue

High-dose therapy and autologous stem cell rescue (ASCR) is the standard of care for chemosensitive relapse, Dr. Zelenetz said. However, he noted that this therapy may also have a role in treatment for patients with only a partial response after second-line chemotherapy.

A study of the CIBMTR registry evaluated chemosensitive patients who had experienced a partial response after second-line therapy. Patients were divided into 2 groups: those who experienced late chemotherapy failure (beyond 12 months) and those with early chemotherapy failure (within the first 12 months). ${ }^{4}$ The investigators found no difference in nonrelapse mortality. Importantly, said Dr. Zelenetz, the risk of progression plateaus at about 18 months after high-dose therapy with ASCR. This translates into a 5-year PFS of $57 \%$ and $48 \%$ for late and early chemotherapy failure, respectively.

"I believe that high-dose therapy and ASCR represents a viable alternative to CAR T cells in the setting of a partial response after second-line chemotherapy," said Dr. Zelenetz. "Additional work needs to be done to determine whether CAR T-cell therapy in this population is superior to high-dose therapy. At this time we do not have any comparative data."

\section{Alternatives to CAR T Cells for Third-Line Relapsed/ Refractory DLBCL}

Alternatives to CAR T-cell therapy in the third-line setting exist, including the recently approved combination of lenalidomide and tafasitamab, an anti-CD19 monoclonal antibody. This combination was tested in the L-MIND study in patients who had relapsed/refractory DLBCL after 1 to 3 prior lines of treatment. ${ }^{5}$ Enrolled patients had to be ineligible for high-dose therapy and ASCR, and those with primary refractory disease were excluded from the study.

The overall median PFS was $>12$ months, said Dr. Zelenetz, but there was a "striking difference" between complete and partial responses, which resulted in an excellent OS for patients with complete response. "The durability of the complete responses is very impressive, and we hope this will hold up in future studies," he said.

\section{Polatuzumab Vedotin}

Another option in the third line is polatuzumab vedotin, which has demonstrated an overall response rate of 
approximately $50 \%$ in relapsed/refractory DLBCL. In combination with rituximab, overall response was also in the range of $50 \%$ to $60 \%{ }^{6}$ Importantly, however, PFS plateaus in approximately $40 \%$ of patients treated with the combination, said Dr. Zelenetz.

A randomized phase II study compared bendamustine/ rituximab versus bendamustine/rituximab + polatuzumab vedotin. ${ }^{7}$ The overall response and complete response rates were clearly better with the addition of polatuzumab to the bendamustine/rituximab backbone, said Dr. Zelenetz, and this translated into improvements in PFS and OS. According to Dr. Zelenetz, however, questions remain regarding the necessity of bendamustine/rituximab in this regimen.

"If you're contemplating using this as a bridge to CAR T cells, bendamustine is toxic to T cells," said Dr. Zelenetz. "If you can avoid the use of a T-cell toxic agent, that is an important consideration."

\section{Mantle Cell Lymphoma}

Finally, the ZUMA-2 trial explored the role of CAR T cells in mantle cell lymphoma. ${ }^{8}$ As Dr. Zelenetz explained, brexucabtagene autoleucel uses the same transgene as axicabtagene ciloleucel, but there is an additional T-cell enrichment step that reduces the risk of transduction of circulating CD19-expressing tumor cells.

ZUMA-2 enrolled 74 patients with relapsed/refractory mantle cell lymphoma and showed a "strikingly low" risk of grade 3 and 4 cytokine-release syndrome, said Dr. Zelenetz.
"It's possible that we've gotten much better at managing this toxicity, or there could be differences in mantle cell lymphoma versus large cell lymphoma," he suggested. "Whatever the reason, the grade 3 and 4 toxicity in the ZUMA-2 trial was clearly less than that reported in ZUMA-1."

With robust responses observed across all subgroups, it is also a highly active agent, said Dr. Zelenetz. The overall response rate was $93 \%$, with $67 \%$ of patients experiencing a complete response and $27 \%$ experiencing a partial response. Median response duration, median PFS, and OS have not been reached at a median follow-up of approximately 1 year. "For a group of patients with relapsed/refractory mantle cell lymphoma, this represents an exciting new treatment option," said Dr. Zelenetz.

The NCCN B-Cell Lymphomas Panel has integrated this agent into the NCCN Guidelines for patients who have had progression or failure on Bruton's tyrosine kinase inhibitors (see page MANT-A in the guidelines, available at NCCN.org). ${ }^{9}$

Disclosures: Dr. Zelenetz has disclosed that he has received grant/research support from BeiGene, Gilead Sciences, Inc., MEl Pharma Inc., and Roche Laboratories, Inc.; has received consulting fees from Adaptive Biotechnologies Corporation, Amgen Inc, BeiGene, Celgene Corporation, Genentech, Inc./ Roche Laboratories, Inc., Gilead Sciences, Inc., Janssen Pharmaceutica Products, LP, Novartis Pharmaceuticals Corporation, and Verastem Oncology and is a Scientific Advisor for AbbVie, Inc, AstraZeneca Pharmaceuticals LP, Genentech, Inc., Gilead Sciences, Inc., MorphoSys AG, and Pharmacyclics.

Correspondence: Andrew D. Zelenetz, MD, PhD, Memorial Sloan Kettering Cancer Center, 1275 York Avenue, New York, NY 10065. Email: zeleneta@mskcc.org

\section{References}

1. Locke FL, Ghobadi A, Jacobson CA, et al. Long-term safety and activity of axicabtagene ciloleucel in refractory large B-cell lymphoma (ZUMA-1): a single-arm, multicentre, phase 1-2 trial. Lancet Oncol 2019;20:31-42.

2. Schuster SJ, Bishop MR, Tam CS, et al. Tisagenlecleucel in adult relapsed or refractory diffuse large B-cell lymphoma. N Engl J Med 2019;380:45-56.

3. Abramson JS, Palomba ML, Gordon LI, et al. Lisocabtagene maraleucel for patients with relapsed or refractory large B-cell lymphomas (TRANSCEND NHL 001): a multicentre seamless design study. Lancet 2020;396: 839-852

4. Shah MA, Kennedy EB, Catenacci DV, et al. Treatment of locally advanced esophageal carcinoma: ASCO guideline. J Clin Oncol 2020;38:2677-2694.

5. Salles G, Duell J, González Barca E, et al. Tafasitamab plus lenalidomide in relapsed or refractory diffuse large B-cell lymphoma (L-MIND): a multicentre, prospective, single-arm, phase 2 study. Lancet Oncol 2020;21: 978-988.

6. Morschhauser F, Flinn IW, Advani $R$, et al. Polatuzumab vedotin or pinatuzumab vedotin plus rituximab in patients with relapsed or refractory nonHodgkin lymphoma: final results from a phase 2 randomised study (ROMULUS). Lancet Haematol 2019;6:e254-265.

7. Sehn LH, Herrera AF, Flowers $C R$, et al. Polatuzumab vedotin in relapsed or refractory diffuse large B-cell lymphoma. J Clin Oncol 2020;38:155-165.

8. Wang M, Munoz J, Goy A, et al. KTE-X19 CAR T-cell therapy in relapsed or refractory mantle-cell lymphoma. N Engl J Med 2020;382:1331-1342.

9. Zelenetz AD, Gordon LI, Abramson JS, et al. NCCN Clinical Practice Guidelines in Oncology: B-Cell Lymphomas. Version 4.2020. Accessed October 26, 2020. Available at NCCN.org 